\title{
Article
}

\section{Large-Area Nanocrystalline Caesium Lead Chloride Thin Films: A Focus on the Exciton Recombination Dynamics}

\author{
Naomi Falsini ${ }^{1,2, *}\left(\mathbb{C}\right.$, , Nicola Calisi ${ }^{3,4}\left(\mathbb{D}\right.$, Giammarco Roini $^{5}$, Andrea Ristori ${ }^{2}$, Francesco Biccari ${ }^{1,2}(\mathbb{D}$,

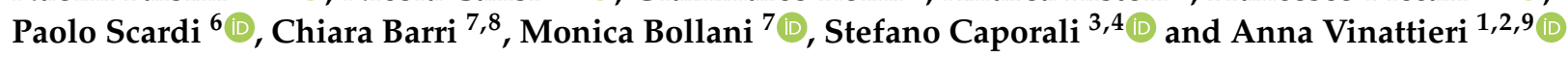 \\ 1 Department of Physics and Astronomy, University of Florence, Via G. Sansone 1, \\ I-50019 Sesto Fiorentino, Italy; francesco.biccari@unifi.it (F.B.); anna.vinattieri@unifi.it (A.V.) \\ 2 European Laboratory for Non-linear Spectroscopy (LENS), University of Florence, Via N. Carrara 1, \\ I-50019 Sesto Fiorentino, Italy; andrea.ristori@unifi.it \\ 3 Department of Industrial Engineering (DIEF), University of Florence, Via S. Marta 3, I-50139 Florence, Italy; \\ nicola.calisi@unifi.it (N.C.); stefano.caporali@unifi.it (S.C.) \\ 4 INSTM-Interuniversity National Consortium for Material Science and Technology, Via Giusti 9, \\ I-50121 Florence, Italy \\ 5 Department of Information Engineering, University of Brescia, Via Branze, 38, I-25123 Brescia, Italy; \\ g.roini@unibs.it \\ 6 Department of Civil, Environmental and Mechanical Engineering, University of Trento, Via Mesiano 77, \\ I-38123 Trento, Italy; Paolo.Scardi@unitn.it \\ 7 Institute of Photonic and Nanotechnology (IFN)-CNR, LNESS Laboratory, Via Anzani 42, I-20133 Como, Italy; \\ chiara.barri@polimi.it (C.B.); monica.bollani@ifn.cnr.it (M.B.) \\ 8 Department of Physics, Polytechnic University of Milan, P.zza Leonardo 32, I-20133 Milano, Italy \\ 9 National Institute for Nuclear Physics (INFN-Firenze), Via G. Sansone 1, I-50019 Sesto Fiorentino, Italy \\ check for \\ updates \\ * Correspondence: naomi.falsini@unifi.it
}

Citation: Falsini, N.; Calisi, N.; Roini, G.; Ristori, A.; Biccari, F.; Scardi, P.; Barri, C.; Bollani, M.; Caporali, S.;

Vinattieri, A. Large-Area

Nanocrystalline Caesium Lead Chloride Thin Films: A Focus on the Exciton Recombination Dynamics. Nanomaterials 2021, 11, 434.

https://doi.org/nano11020434

Academic Editors: Jan Valenta and Juan P. Martínez Pastor

Received: 30 December 2020

Accepted: 5 February 2021

Published: 9 February 2021

Publisher's Note: MDPI stays neutral with regard to jurisdictional claims in published maps and institutional affiliations.
Abstract: Caesium lead halide perovskites were recently demonstrated to be a relevant class of semiconductors for photonics and optoelectronics. Unlike $\mathrm{CsPbBr}_{3}$ and $\mathrm{CsPbI}_{3}$, the realization of high-quality thin films of $\mathrm{CsPCl}_{3}$, particularly interesting for highly efficient white LEDs when coupled to converting phosphors, is still a very demanding task. In this work we report the first successful deposition of nanocrystalline $\mathrm{CsPbl}_{3}$ thin films $(70-150 \mathrm{~nm})$ by radio frequency magnetron sputtering on large-area substrates. We present a detailed investigation of the optical properties by high resolution photoluminescence (PL) spectroscopy, resolved in time and space in the range $10-300 \mathrm{~K}$, providing quantitative information concerning carriers and excitons recombination dynamics. The PL is characterized by a limited inhomogeneous broadening ( $15 \mathrm{meV}$ at $10 \mathrm{~K})$ and its origin is discussed from detailed analysis with investigations at the micro-scale. The samples, obtained without any post-growth treatment, show a homogeneous PL emission in spectrum and intensity on large sample areas (several $\mathrm{cm}^{2}$ ). Temperature dependent and time-resolved PL spectra elucidate the role of carrier trapping in determining the PL quenching up to room temperature. Our results open the route for the realization of large-area inorganic halide perovskite films for photonic and optoelectronic devices.

Keywords: inorganic halide perovskites; $\mathrm{CsPbCl}_{3}$; thin films; sputtering; high resolution photoluminescence

\section{Introduction}

Research on new materials has recently focused on halide perovskites as highly promising semiconductors for advanced photonic and optoelectronic applications [1,2] Indeed, caesium lead halide perovskites, described by the formula $\mathrm{CsPbX}_{3}(\mathrm{X}=\mathrm{Cl}, \mathrm{Br}$, I), are excellent active materials for coherent and incoherent light sources, sensors and innovative solar cells [3-5]. Their relevant electronic and optical properties (direct band gap, fine gap tunability by changing the halogen and alloying, high carrier mobility, defect tolerance, etc.) and, in particular, their higher thermal and chemical stability with respect 
to hybrid organic-inorganic perovskites [6-8], make them attractive for the development of highly performing light sources and for the integration in photonic structures. Among these fully inorganic perovskites, $\mathrm{CsPbCl}_{3}$ (band gap about $3.1 \mathrm{eV}$ at room temperature [9]) is optimal for the development of light emitters in the blue spectral range, as a substitute of nitride-based materials, and it is the most suitable for the realization of white LEDs, coupled to a converting phosphor.

So far, solution-based techniques represent the most common route for the deposition of both organic-inorganic and fully inorganic perovskite films $[7,10,11]$. However, the main problems of these techniques are the limited scalability and/or the inhomogeneity of the deposited material, negatively affecting the large-scale production and external quantum efficiencies for light emission. Usually, to overcome the reduced material quality, the addition of chemicals is required in combination with post-deposition annealing. Moreover, to the best of our knowledge the deposition of $\mathrm{CsPbCl}_{3}$ films through dissolution of its precursor salts is not achievable. Different strategies for the synthesis of $\mathrm{CsPbCl}_{3}$ have been reported in literature: bulk crystals by the Bridgman method [12]; nanocrystals by high temperature solvent [9] and similar methods; thin films by evaporation [13-17]. It is worth mentioning that the possibility of realizing large-area compact films of nanometric thickness is relevant for the scalability of innovative devices. Moreover, the use of a technique like the sputtering discussed in this work, that allows for multilayers deposition (i.e., active material, electron/hole transport layers, metallic coatings, etc.) in a controlled atmosphere, over different substrates, opens the route for a wide set of applications in the field of photonics and optoelectronics, including the realization of metasurfaces.

In this work, following our recent paper on $\mathrm{CsPbBr}_{3}$ [18], we have obtained the first and successful deposition of nanocrystalline $\mathrm{CsPCl}_{3}$ thin films by radio frequency (RF) magnetron sputtering. We present a detailed study of a set of samples with film thickness in the range 70-150 nm deposited on two different kinds of substrates. Beside morphological, structural and compositional analysis, we investigated the material optical properties by high resolution photoluminescence (PL) spectroscopy, resolved in time and space in a wide temperature range (10-300 K), providing quantitative information concerning the recombination dynamics of carriers and excitons. Our results demonstrate the macroscopic homogeneity of the $\mathrm{CsPbCl}_{3}$ thin films on large sample areas, which is hardly achievable, especially with a single step deposition, without the addition of specific chemicals and without post-growth treatments.

\section{Materials and Methods}

Several thin films of $\mathrm{CsPbCl}_{3}$ were deposited by RF magnetron sputtering onto two properly cleaned different substrates: soda lime glass (SLG) and amorphous quartz slides. The magnetron sputter used for sample preparation is a HEX system (Korvus Technology Ltd., Newington, UK) equipped with an RF source, a rotating sample holder to ensure the uniformity of the deposited film and a gravimetric microbalance to set the film thickness. The deposition was obtained in a non-reactive Argon atmosphere with a gas flow of $20 \mathrm{sccm}$ (deposition rate $0.05 \mathrm{~nm} \mathrm{~s}^{-1}$ ). The sputtering of $\mathrm{CsPbCl}_{3}$ was obtained by a homemade target fabricated by mixing $\mathrm{CsCl}$ and $\mathrm{PbCl}_{2}$ precursor salts (Merck KGaA, Darmstadt, Germany) in equal molar ratio. The salts were dried in oven at $120^{\circ} \mathrm{C}$ overnight and ground, by using a mixer mill (model MM400, Retsch, Haan, Germany), to obtain a uniform mixing. The mixture was then pressed at $11.5 \mathrm{MPa}$ while the system was heated at $160{ }^{\circ} \mathrm{C}$ for the sintering of the powder. Further details on the sputtering procedure are reported in ref. [18].

Samples were characterized by scanning electron microscopy (SEM), atomic force microscopy (AFM), X-ray photoelectron spectroscopy (XPS) and X-ray diffraction (XRD) to assess morphology, crystalline structure and stoichiometry. Details concerning the used setups for these investigations are provided in the Supplementary Material.

XRD spectra were collected on a X'Pert diffractometer (Panalytical, Malvern, UK) equipped with $\operatorname{CoK} \alpha$ X-ray source $(40 \mathrm{kV}, 40 \mathrm{~mA})$, with a polycapillary optics in the 
primary beam (with $1 \mathrm{~mm}$ in equatorial direction and $10 \mathrm{~mm}$ height) and flat graphite crystal analyzer in the secondary beam, before the proportional counter. The grazing incidence measurements were made at a grazing incidence angle of $0.9^{\circ}$ (actual incidence depends on the sample, as glass substrates are never perfectly flat). Beam divergence, as provided by the polycapillary lens, is $0.3^{\circ}$. Phase identification was based on the ICDD PDF-4+ database. All data, conventional $\theta / 2 \theta$ and grazing incidence, were collected with a sampling step of $0.04^{\circ}$ and counting time of $20 \mathrm{~s}$. The XRD card matching the peak positions is \#18-0366 of the ICDD PDF-4+ database (Tetragonal CsPbCl 3 , Space Group P4mm (99), unit cell parameters $\mathrm{a}=\mathrm{b}=5.584 \AA, \mathrm{c}=5.623 \AA$ ), corresponding to a tetragonal crystal phase.

A Cary 300 spectrophotometer (Agilent, Santa Clara, CA, USA) equipped with a PELA-1050 integrating sphere (Labsphere, North Sutton, NH, USA) was used for the transmittance spectra of the samples at room temperature.

The PL of the deposited $\mathrm{CsPbCl}_{3}$ films was characterized by two different spectroscopy setups for detection at the macro and micro-scale. Most part of PL experiments was realized in a macro-PL configuration (laser spot diameter $\approx 100 \mu \mathrm{m}$ ) in a quasi-backscattering geometry, keeping the samples in a closed cycle cryostat and varying the temperature in the range $10-300 \mathrm{~K}$. The excitation intensity was at maximum $10 \mathrm{~W} / \mathrm{cm}^{2}$. A frequencydoubled mode-locked ps Ti:Sapphire laser, operating at $81.3 \mathrm{MHz}$ repetition rate with $1.2 \mathrm{ps}$ pulses, was used for time-integrated (TI) and time-resolved (TR) experiments: the excitation photon energy was varied in the range 3.3 to $3.45 \mathrm{eV}$. The fourth harmonic $(266 \mathrm{~nm})$ of a Q-switched Nd:YAG laser was used for excitation at $4.67 \mathrm{eV}$ (repetition rate $20 \mathrm{KHz}$, 300 ps pulse duration). The PL signal was spectrally dispersed by a monochromator providing a spectral resolution of $1 \mathrm{meV}$ and detected by a charge coupled device (CCD) detector (DU420-BU, Andor, Belfast, UK) for TI PL spectra or a synchroscan streak camera (C5680, Hamamatsu, Shizuoka, Japan) for TR measurements (time resolution $\approx 5 \mathrm{ps}$ ). Transmittance spectra in the temperature range $10-300 \mathrm{~K}$ were acquired simultaneously to PL by means of a white lamp.

For micro-PL experiments, the sample was kept at $10 \mathrm{~K}$ in a low-vibration ST-500 continuous He-flow cryostat (Janis, Lake Shore Cryotronics, Inc., Westerville, OH, USA) which in turn was mounted on an $x-y$ translation stage (Physik Instrumente, Karlsruhe, Germany) for scanning the sample surface. The luminescence was collected by a home-made confocal microscope setup equipped with an infinity corrected $50 \times$ NUV objective (Mitutoyo, Neuss, Germany, 378-818-6, NA = 0.42). The luminescence was spectrally dispersed and detected using a SP2300i spectrograph (Acton, Teledyne Princeton Instruments, Krailing, Germany) equipped with two $1200 \mathrm{gr} / \mathrm{mm}$ gratings blazed at $350 \mathrm{~nm}$ and $750 \mathrm{~nm}$, and an Acton Pixis 100F Si CCD (Teledyne Princeton Instruments, Krailing, Germany). The spatial resolution of the system is about $700 \mathrm{~nm}$, while the spectral resolution is about $250 \mu \mathrm{eV}$. The excitation source was provided by a frequency-doubled mode-locked Ti:Sapphire tunable laser (Tsunami, Spectra Physics, Santa Clara, CA, USA, 700-900 nm spectral range, 200 fs pulse duration, $12.2 \mathrm{~ns}$ pulse period). The experiments were performed exciting the samples with photons of $3.40-3.45 \mathrm{eV}$ and an excitation intensity at maximum of $100 \mathrm{~W} / \mathrm{cm}^{2}$.

\section{Results}

\subsection{Samples Characterization at Room Temperature}

The samples investigated in this work are listed in Table $1: \mathrm{CsPCl}_{3}$ films of different thickness (70 and $150 \mathrm{~nm}$ ) were realized on SLG and amorphous quartz substrates.

Table 1. Name, thickness and substrate type of investigated samples.

\begin{tabular}{ccc}
\hline Sample & Average Thickness $\mathbf{( n m )}$ & Substrate \\
\hline A & 70 & SLG \\
B & 70 & Quartz \\
C & 150 & SLG \\
D & 150 & Quartz \\
\hline
\end{tabular}


Highly uniformity and homogeneity over the substrate is always found at the macroscale, as shown in the photograph of Figure 1a. The morphological characterization at the micro-scale is reported in Figure $1 b-d$, where a SEM micrograph and AFM analysis are respectively shown. They point out the presence of a fairly compact network of nanocrystals with average lateral size of $50 \mathrm{~nm}$ and average thickness of $40 \mathrm{~nm}$ just on the top of the substrate. Larger crystals (quite well isolated one from the other), up to several hundred nanometers size and with a height around $100 \mathrm{~nm}$, are also found. Such values have been extracted by analyzing AFM profiles in different regions of the sample with an estimated uncertainty of $\pm 10 \%$. Hereafter, we identify with NC the network of nanocrystals and with MCs the sub-micrometer size larger crystals. Similar morphology is found from SEM investigation for samples differing in thickness or substrate.
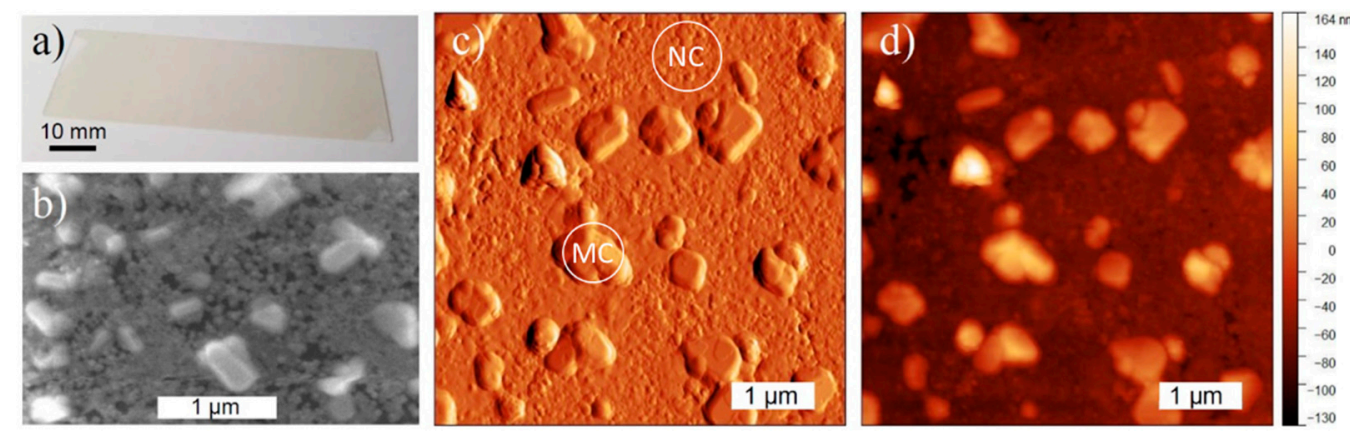

Figure 1. (a) Typical photograph of a $70 \mathrm{~nm}$ thick $\mathrm{CsPbCl}_{3}$ sample; (b) SEM micrograph of sample A. (c,d) AFM amplitude and topography maps of a $5 \times 5 \mu \mathrm{m}^{2}$ area of sample A. The circles with $\mathrm{NC}$ and MC indicate, as an example, regions with nanocrystals and sub-micrometer size larger crystal, respectively.

Phase identification was obtained via grazing angle incidence and $\theta / 2 \theta$ XRD investigation. The XRD analysis (for details concerning the experimental apparatus see ref. [19]) clearly indicates the presence of the $\mathrm{CsPbCl}_{3}$ crystalline tetragonal phase (Figure 2a), as expected at room temperature [20,21], with a significant h00 fiber orientation which is commonly observed in our samples. The XRD results are compatible with the picture of a thin film made of two distinct perovskite fractions. In $\theta / 2 \theta$ only (100) and (200) reflections are detected, while in grazing angle incidence other peaks are reported (Figure $2 \mathrm{a}$ ). The broad peak at 26 degrees comes from the amorphous substrate. The $\theta / 2 \theta$ diffraction pattern shows the signal from crystalline grains with (hkl) atomic planes parallel to the surface: besides the broad signal from the large fraction of the X-ray beam going through the thin film and being diffracted by the amorphous substrate underneath, the two strong h00 lines in the $\theta / 2 \theta$ pattern are given by large and presumably (fibre) textured grains. In the grazing incidence condition, instead, the large crystals contribute much less, except for the 100 line. AFM and SEM images (Figure 1) show that the large grains, better crystallized to the point that in some sample regions they even assume a geometrical shape typical of single crystals, have a large spread of orientations, thus explaining the presence of a still intense 100 peak even in grazing condition. The remaining part of reflections observed in grazing incidence, however, are sensibly broader and belong to planes with different orientations, likely originating from the (nano)polycrystalline fraction, which appears randomly oriented or just weakly textured. Even though, given the instrumental conditions, it is not possible to precisely determine the size of the domains, the observed broadening is compatible with perovskite domain sizes of the order of several tens of nanometers in agreement with the AFM and SEM images. 

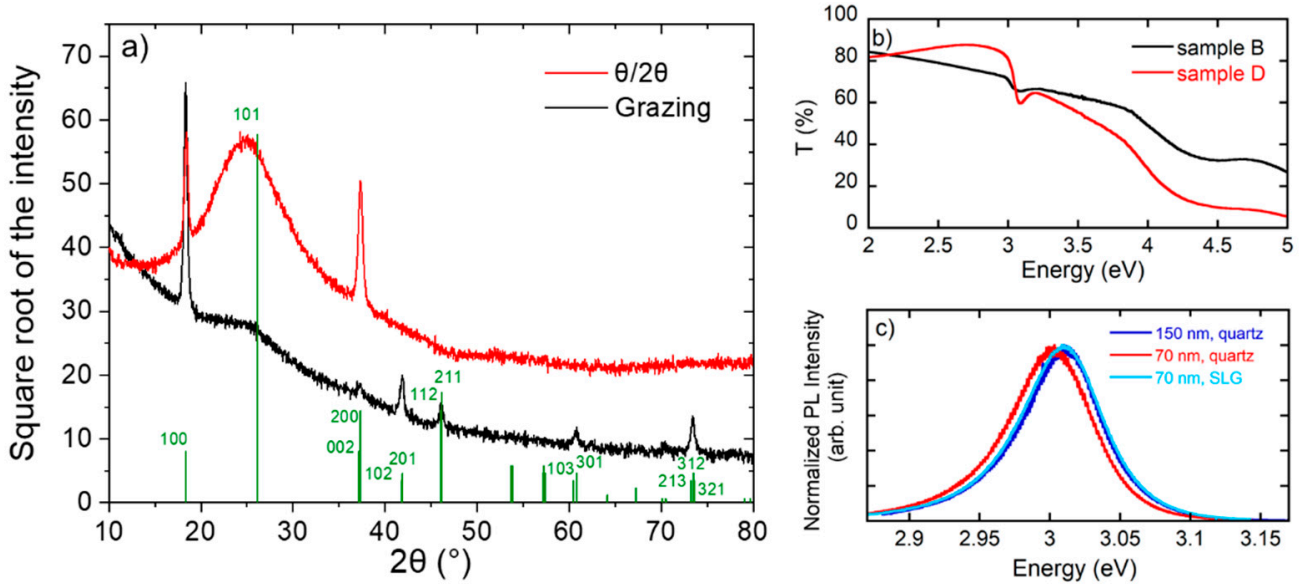

Figure 2. (a) XRD spectra of sample B in $\theta / 2 \theta$ (red line) and grazing angle incidence (black line) configuration. The bars mark positions of the identified tetragonal phase. (b) Transmittance spectra of samples B (in black) and D (in red) at room temperature. (c) Normalized macro-PL spectra at room temperature comparing samples differing in thickness and substrate.

XPS spectra (Figure S1) were used to estimate the stoichiometry of the film surface. The binding energy values of caesium, lead and chlorine are in accordance with the literature data [22]. Literature sensitivity factors were applied and the atomic ratio of the elements are reported in Table 2 for different substrate and same thickness. They show a lead excess and a chlorine lack respect to the expected values [23]. It is worth mentioning that the discrepancy between the measured stoichiometry and the expected one can be ascribed to the peculiar nature of the XPS investigation that provides information only on a few nanometers below the surface. Presence of oxygen and carbon can be evidenced by XPS spectra (Figure S1). While adventitious carbon contamination, appearing as soon as samples are exposed to atmosphere, can be claimed as carbon source, oxygen is more likely related to the presence of metallic oxides, as demonstrated by the presence of a peak at $529 \mathrm{eV}$ (Figure S1). Moreover, we observe that the intensity of the oxygen peak is modestly reduced by argon sputtering which is consistent with the presence of compounds formed during film growth rather than as a result of post deposition atmosphere exposure. Unambiguous identification of these minor compounds is out of the scope of the present work, however, lead oxides like $\mathrm{PbO}$ or $\mathrm{PbO}_{2}$, are the most reasonable candidates. Similar results are found for all the investigated samples.

Table 2. XPS experimental atomic ratio and expected values for samples C and D.

\begin{tabular}{cccc}
\hline Element & Glass (Sample C) & Quartz (Sample D) & Expected \\
\hline $\mathrm{Cs}$ & $20 \%$ & $19 \%$ & $20 \%$ \\
$\mathrm{~Pb}$ & $30 \%$ & $30 \%$ & $20 \%$ \\
$\mathrm{Cl}$ & $50 \%$ & $51 \%$ & $60 \%$ \\
\hline
\end{tabular}

Transmittance spectra at room temperature are shown in Figure $2 \mathrm{~b}$ for $\mathrm{B}$ and $\mathrm{D}$ samples. The transitions at $4.4 \mathrm{eV}$ and $3 \mathrm{eV}$ are evident in absorption, as expected and reported in previous works for caesium lead chloride films and single crystals [14,24,25]. In particular, the narrow dip at $3 \mathrm{eV}$ is ascribable to the fundamental excitonic resonance, characteristic of $\mathrm{CsPbCl}_{3}[14,24,25]$.

\subsection{Photoluminescence Study}

The main focus of our work concerns PL properties to validate the good optical quality of the material. Different PL experiments were performed changing the spatial resolution of the PL setup, from $1 \mu \mathrm{m}$ to $100 \mu \mathrm{m}$. A comparison of the PL emission at room temperature is shown in Figure 2c for samples differing in thickness/substrate (PL spectra at low $\mathrm{T}$ 
are shown in Supplementary Information in Figure S2). The PL line shape does not show relevant changes; only a slight shift of the PL peak energy, likely related to the different strain between the perovskite film and the substrate. More significant is the PL intensity change from spot to spot spanning a sample area of $\sim 10 \mathrm{~cm}^{2}$ (Figure S2). While most of the spectra change in intensity of the order of $30 \%$, a few cold spots are found with a reduced PL intensity.

\subsubsection{Photoluminescence at Macro and Micro-Scale}

To gain insights in the PL excitonic features low temperature spectra have been measured at micro- and macro-scale. A typical macro-PL spectrum of a $\mathrm{CsPbCl}_{3}$ sample on SLG substrate at $10 \mathrm{~K}$ is displayed in Figure 3a and compared with the high-resolution transmittance spectrum detected in the same sample spot. At $10 \mathrm{~K}$ the PL spectrum shows a dominant emission ( $\alpha$-band) peaked at $2.97 \mathrm{eV}$, in agreement with literature data on $\mathrm{CsPbCl}_{3}$ bulk and nano-crystals and thin films [26-28], and a less intense higher energy band ( $\beta$-band) peaked at $\approx 3.02 \mathrm{eV}$ which has also been reported in previous works $[28,29]$. At $10 \mathrm{~K}$ both bands, with a full width half maximum of about $15 \mathrm{meV}$ comparable to single crystals [26], correspond to two resonances in the transmittance spectrum (Figure 3a) with a similar Stokes shift $\leq 5 \mathrm{meV}$, indicating an overall high quality of the emission that arises from two excitonic recombinations.
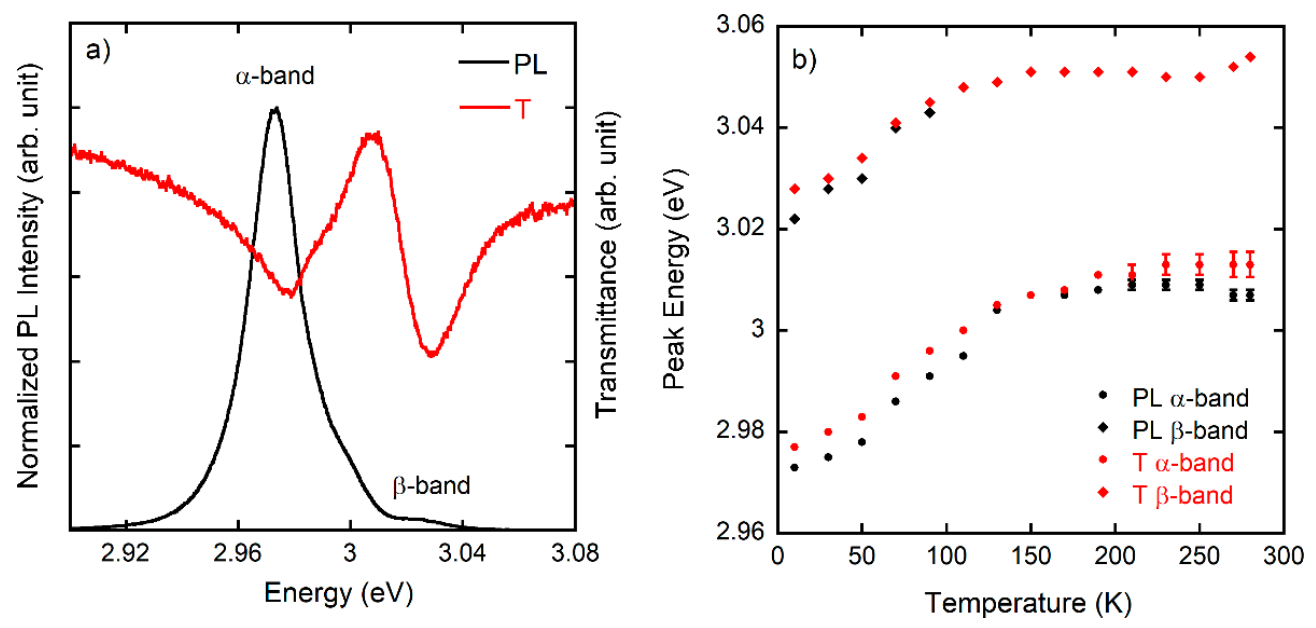

Figure 3. (a) Macro-PL (black curve) and transmittance (red curve) spectrum on sample A at $10 \mathrm{~K}$ after excitation at $4.67 \mathrm{eV}$. (b) Emission energy of the maximum of the PL for the $\alpha$ and $\beta$-band along with the corresponding minima of the transmittance spectra as a function of temperature for sample A.

Figure $3 \mathrm{~b}$ displays the temperature dependence of the PL peak energy of both bands and transmission resonances, confirming that excitonic features are observed up to room temperature. A decrease of the Stokes shift between PL and transmission is observed from 10 to $150 \mathrm{~K}$. The macro-PL spectra as a function of temperature (T) (Figure S3) show, as expected, a blue shift of the spectrum and a quenching of the emission; moreover, in the $\alpha$-band a structure appears at higher energy ascribable to a free exciton (FE) recombination around $100 \mathrm{~K}$ that becomes dominant as the temperature increases.

Power dependent PL spectra at $10 \mathrm{~K}$ reveal a linear dependence of the emission as expected for geminate (excitonic) recombination of carriers or when the radiative recombination prevails over the non-radiative one.

At low temperature macro-PL spectra show negligible sample inhomogeneity in terms of PL intensity of each band and peak energy shift. To deeply investigate the sample inhomogeneity so as to provide information on the origin of the inhomogeneous broadening (IB) we performed micro-PL experiments with a spatial resolution $\leq 1 \mu \mathrm{m}$. Typical results are shown in Figure $4 a$ where we compare spectra in different points. 
Micro-PL spectra highlight a finer structure of the $\alpha$-band with contributions in the low energy side and a shoulder in the high energy side, reported in Figure 4a. According to literature [27], the shoulder on the low energy side of $\alpha$-band could come from a free exciton phonon replica or, more likely, to a bound exciton in a deeper defect. The shoulder present in the high energy side of the $\alpha$-band is, instead, related to the $\beta$-band (see Pearson correlation map of Figure $4 \mathrm{~b}$ ). Hereafter the peaks of the two bands are indicated as $\mathrm{P}_{\alpha}$ and $\mathrm{P}_{\beta}$. It clearly appears that micro-PL spectra have IB similar to the one of the macro-PL spectrum of Figure 3a; differences are observed in the relative emission intensity of $\alpha$ and $\beta$-band (Figure 4a). It has to be noted that no normalization has been applied to the spectra of Figure 4a so that the emission of $\beta$-band is constant in the investigated sample region. Figure $4 \mathrm{~b}$ reports the Pearson correlation map (in the Pearson correlation map each pixel, identified by two energy values, $E_{1}$ and $E_{2}$, is calculated correlating the PL intensity at $E_{1}$ with the one at $E_{2}$ over the entire ensemble of spectra (2500 in our case) in the micro-PL map) in a $25 \times 25 \mu \mathrm{m}^{2}$ sample area, showing that the emission of $\alpha$ and $\beta$ bands are spectrally uncorrelated, therefore excluding the attribution of the $\beta$-band to an excited state of the $\alpha$-band. A comparison among micro-PL maps, AFM and SEM images (Figure $1 b-d$ ) and results from XRD spectrum (Figure 2a) suggests the attribution of $\alpha$-band to MCs and $\beta$-band to NC. In Figure $4 c, d$ the PL intensity variations for the $\alpha$ and $\beta$-band contributions are reported, respectively. $\beta$-band is constant in intensity in sample macro-areas (hundreds of $\mu \mathrm{m}^{2}$ ), while intensity fluctuations at most of a factor 3 are observed for $\alpha$-band. The PL peak energy variation in the sample is at maximum $\pm 3 \mathrm{meV}$ for $\mathrm{P}_{\alpha}$ (Figure S4) and less than $1 \mathrm{meV}$ for $\mathrm{P}_{\beta}$. No correlation emerges between the emission peak energy and PL intensity, as from the comparison between the maps of Figure S4 and Figure 4c. The micro-PL shows also that, for all the investigated samples, the largest contribution to the IB of PL at the macro-scale does not come from disorder detectable at the micro-scale: in fact, the spatial fluctuation of the PL peak energy (average value $2.969 \mathrm{eV}$ and standard deviation $1.8 \mathrm{meV}$ ) accounts for $\approx 10 \%$ of the overall IB, the remaining part being related to a sub-micrometer disorder attributable to the size inhomogeneity of the MCs. Moreover, we exclude that the major part of the broadening comes from a homogeneous contribution given the long dephasing time measured in inorganic lead halide perovskites [30].
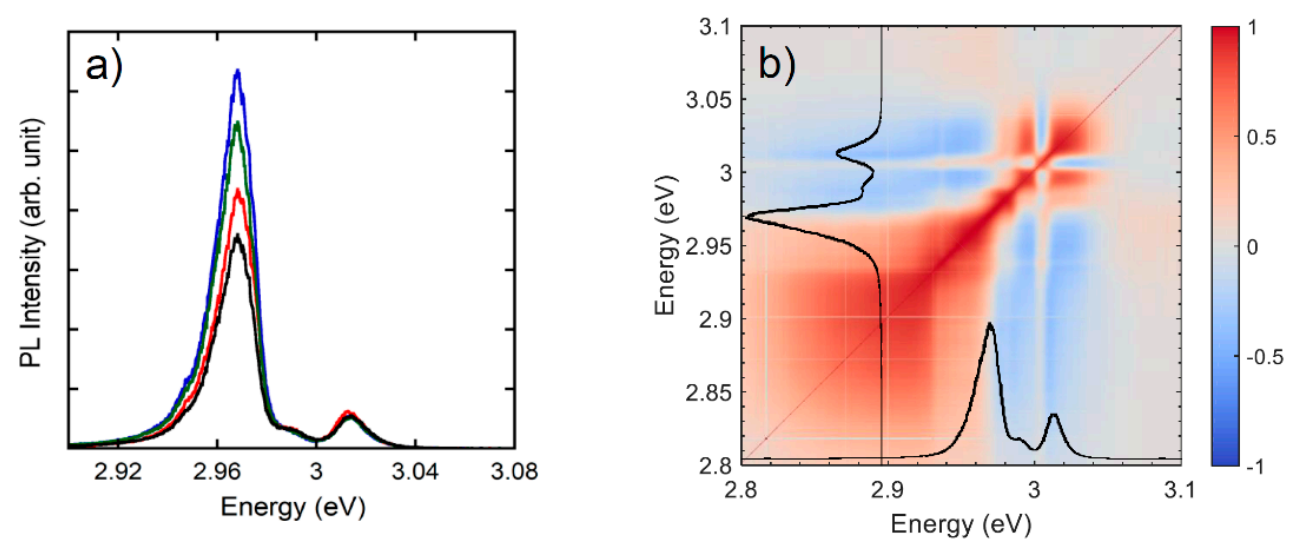

Figure 4. Cont. 

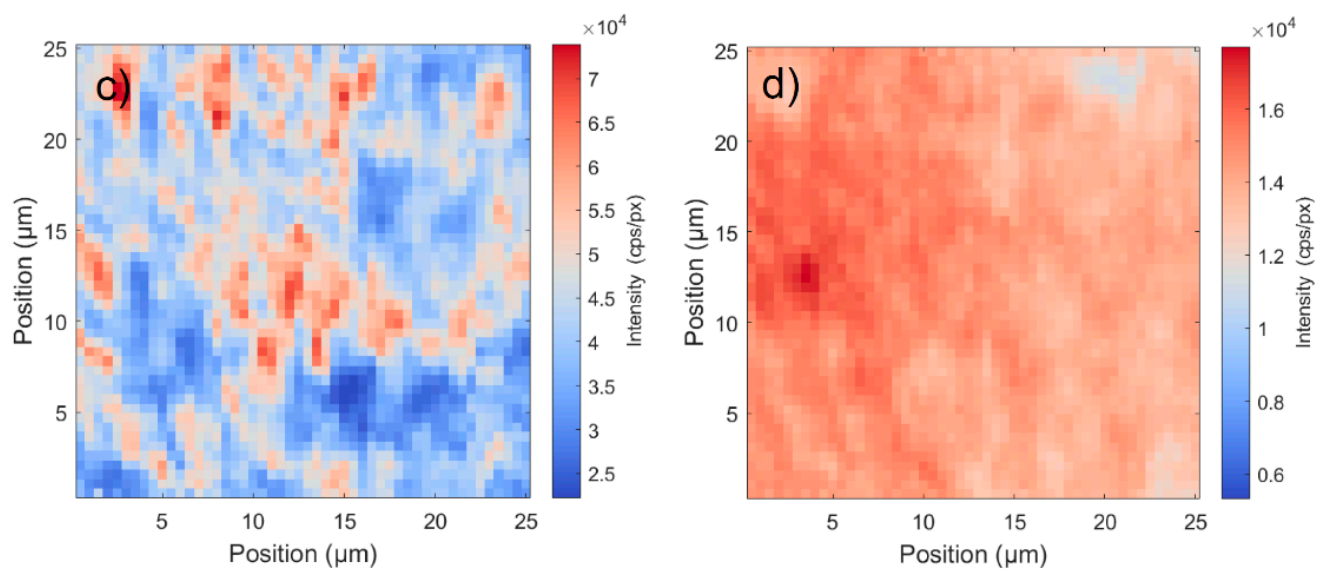

Figure 4. (a) Micro-PL spectra at $10 \mathrm{~K}$ for sample A acquired in different sample spots spanning a $25 \times 25 \mu \mathrm{m}^{2}$ area. (b) Pearson correlation map in the same sample area. The black lines correspond to the spatially integrated PL spectra in the same sample area. (c,d) PL intensity for $P_{\alpha}$ and $P_{\beta}$, respectively, in the same sample area.

\subsubsection{Time-Resolved Photoluminescence}

In order to clarify the nature of the recombination of $\alpha$ and $\beta$-band, we performed TR spectra at different temperatures (from 10 to $280 \mathrm{~K}$ ) after picosecond excitation at $3.45 \mathrm{eV}$ with an intensity of $10 \mathrm{~W} / \mathrm{cm}^{2}$, corresponding to a photon flux of $\sim 4 \times 10^{11}$ photons $/ \mathrm{cm}^{2}$. Results at $14 \mathrm{~K}$ are reported in Figure 5. A typical streak camera image is shown in Figure 5a, from which TR spectra and decays are extracted (Figure 5b-d). The TR spectra at different delay times are shown in Figure $5 b$ on a log scale: all spectra, at each time delay, exhibit an exponential thermal tail whose effective time dependent temperature can be evaluated assuming the Boltzmann distribution for the carriers [31,32]. In this case the initial carrier temperature around $140 \mathrm{~K}$ progressively decreases down to $80 \mathrm{~K}$ (see Figure S5), maintaining a value higher than the lattice temperature for a long time, as already observed in $\mathrm{CsPbBr}_{3}$ [32]. After a fast rise the population thermalizes and, as a consequence, all the states around $\mathrm{P}_{\alpha}$ (in the range 2.960-2.990 eV) decay with the same time constant. Instead, a strong difference of the PL lifetime is observed at $\mathrm{P}_{\alpha}$ and $\mathrm{P}_{\beta}$ (Figure $5 d$ ), and the TR spectra of Figure $5 b$ indicate a substantial independence of the two bands recombination with a lack of population transfer between $\beta$ and $\alpha$ bands, confirming the absence of correlation of the two (Figure $4 b$ ).
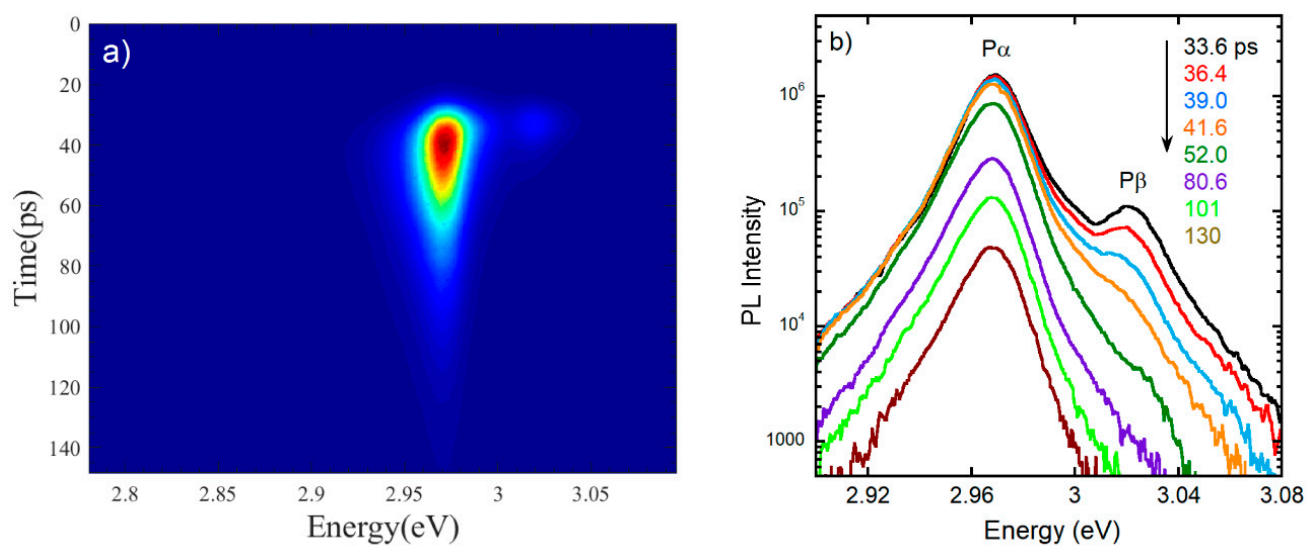

Figure 5. Cont. 

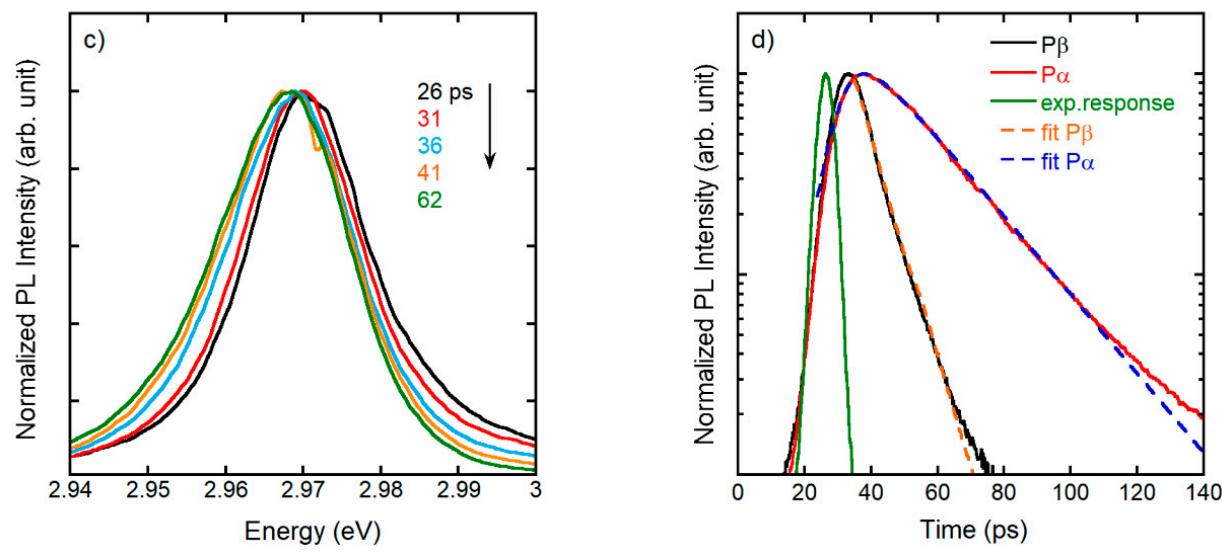

Figure 5. (a) Typical streak camera image of the time-resolved (TR) macro-PL at $14 \mathrm{~K}$ for sample A; (b) TR PL spectra extracted from a); (c) Normalized TR spectra showing the transfer of population from higher to lower energy states at increasing time delay; (d) PL decays (continuous line) extracted from (a) at peaks energy of $\mathrm{P}_{\alpha}$ (in red) and $\mathrm{P}_{\beta}$ (in black) along with the experimental time response (in green). The fit functions (dashed lines) are shown superimposed to the experimental PL decays.

The initial change in the spectral shape of $\alpha$-band, due to thermalization, is shown in Figure $5 \mathrm{c}$ where we can easily notice that by increasing the time delay the population transfers from higher energy, corresponding to FE, to lower energy states, i.e., to localized or bound exciton states. After nearly 60 ps the PL decreases in intensity without further change in its shape, attesting the achievement of the thermalization. It is to remark that the TI spectrum at low $T$ has a peak energy at $P_{\alpha}$, corresponding to a bound exciton recombination. Further evidence of such attribution comes from temperature-dependent spectra showing increased contribution of FE at higher temperature (Figure S3).

The PL time evolution at $P_{\alpha}$ and $P_{\beta}$, at short times, and the corresponding fits are shown in Figure $5 \mathrm{~d}$, along with the experimental time response. In the fastest time scale, up to $100 \mathrm{ps}$, a single exponential decay with a time constant $\tau_{1}$ can substantially describe the PL time evolution. Each fitting curve is obtained as convolution between the experimental time response and the PL decay function, using an exponential rise and decay; the time constants for $P_{\alpha}$ are $\tau_{\text {rise }} 4$ ps and $\tau_{1} 22$ ps, while for $P_{\beta} \tau_{\text {rise }}$ is resolution limited and $\tau_{1}$ is $7 \mathrm{ps}$. TR PL data for all the investigated samples do not show remarkable changes in the time constant for $\alpha$ and $\beta$-band. It is also worth to remark that no change into the PL dynamics was observed by changing the excitation power over two orders of magnitude, between 1 and $100 \mathrm{~W} / \mathrm{cm}^{2}$.

\section{Data Analysis and Discussion}

Our TR data prove that $\alpha$ and $\beta$ emissions, which are present in all the investigated samples with similar features in terms of spectral line shape, relative intensity and time evolution, do not originate from a relaxation of higher energy ( $\beta$-band) to lower energy states ( $\alpha$-band). This is an additional confirmation that they do not originate from the same spatial region in agreement with results of Figure $4 \mathrm{~b}$. Moreover, in this context, the significant faster decay of $\mathrm{P}_{\beta}$ respect to $\mathrm{P}_{\alpha}$ is naturally explained by the major role played by surface state recombination in the NC as expected by the increased surface over volume states when the crystal size decreases [33].

In the comparison of our PL results with literature data on single crystal and amorphous films, it turns out that the main peak $\mathrm{P}_{\alpha}(\approx 2.97 \mathrm{eV}$ at $10 \mathrm{~K})$ corresponds to a localized exciton recombination of $\mathrm{CsPbCl}_{3}[26,27]$.

More controversial is the assignment of the emission at $\approx 3.02 \mathrm{eV}$ ( $\beta$-band) already observed in single crystals, nanocrystals and amorphous films. This band is reported in previous works with different attributions; Ito et al. assign the $\beta$-band to the $2 \mathrm{~S}$ excitonic transition [29], whereas Lohar et al. to a bound exciton [28]. Kondo et al., performing experiments at $77 \mathrm{~K}$ and high excitation density $\left(>10 \mathrm{~kW} / \mathrm{cm}^{2}\right)$ observes a band at $2.97 \mathrm{eV}$ 
with a superlinear behavior claimed as stimulated emission, while the FE emission is assigned to a band at $\approx 3.03 \mathrm{eV}$ [34]. Our data exclude all the previous assignments of the $\beta$-band. Indeed, concerning the $2 S$ recombination we have proven in TR spectra and in micro-PL maps that the $\beta$-band has no correlation in time and space with $P_{\alpha}$. Moreover, the power dependence of the PL intensity and excitation density used in our experiment exclude the presence of stimulated emission in the spectra. It has to be noted that $\beta$-band cannot be attributed to the precursor salts [35,36]. Moreover, the analysis of micro-PL maps (Figure 4) exclude any intermixing between $\alpha$ and $\beta$-band, corresponding respectively to the MCs and to the NC with a lack of "communication" between the two kind of emitters. We remark that such spectral features are always found in our samples, even though with different intensity, indicating that they are related to the growth conditions. To explain the different energies of the $\alpha$ and $\beta$-band several effects can be invoked: first of all, given the size of NC and MCs and the exciton Bohr radius of $2.5 \mathrm{~nm}$ [9], quantum confinement effects must be excluded provided that they are relevant only for NC with dimensions smaller than $10 \mathrm{~nm}$. A possible origin could be related to the presence of a different strain between the substrate and $\mathrm{NC}$ and MCs, that show, however, an equal temperature dependent behavior (Figure 3b). We can envisage also two other possible origins of the different bands: a change in the stoichiometry of $\mathrm{Cs}, \mathrm{Pb}, \mathrm{Cl}$ [37] or different crystalline phase of $\mathrm{CsPbCl}_{3}$ in the MCs and NC, in analogy with reports on hybrid perovskites [38-40]. Two recent papers [41,42] highlighted structural changes crossing between NC and MCs and therefore it is very likely to suppose that the different energies of the $\alpha$ and $\beta$ bands reflect such changes. Presently, we cannot experimentally distinguish between the previous hypotheses about the origin of the $\beta$-band: however, it must be pointed out that our data clearly show the formation of extended electronic states as certified by the excitonic resonances of Figure 3.

A relevant aspect of our work concerns the temperature-dependent TR data and, in particular, the relative contribution of radiative and non-radiative recombination, providing quantitative estimate of the PL yield. In Figure 6a the PL time evolution at $P_{\alpha}$ for different temperatures is reported, over an extended range with respect to Figure $5 \mathrm{~d}$, showing a non-exponential behavior: for each temperature the decay can be nicely fitted with two exponentials with time constants $\tau_{1}$ and $\tau_{2}$, and the results are plotted in Figure $6 \mathrm{~b}$. The fast decay time $\tau_{1}$, after an initial rise at low temperature $(T)$, decreases to smaller values, but the overall variation in the range $10-300 \mathrm{~K}$ is less than a factor 5 ; similar limited variation is observed for $\tau_{2}$; the small increase of both time constants observed for $T>150 \mathrm{~K}$ can be ascribed to the second order phase transition reported around this temperature for $\mathrm{CsPbCl}_{3}$ [43-45]. The fast initial decay time constant agrees with results of [46]. In Figure $6 \mathrm{c}$ we report different Arrhenius plots extracted from the PL intensity as a function of $T$ that gives account of the PL quenching. In Figure $6 \mathrm{c} I_{T I}$ indicates the PL intensity of the $\alpha$-band integrated in time and over all the spectrum of the band itself, $I\left(E_{P \alpha}\right)$ is the TI PL intensity at $P_{\alpha}$ peak energy $E_{P \alpha}$, and $I\left(E_{P \alpha}, 0\right)$ is the maximum of intensity at $E_{P \alpha}$ as obtained from the TR spectra at different temperatures. The quantity $F\left(E_{P \alpha}\right) / \tau_{R}$ will be defined in the following. Similar trends concerning the temperature dependence of the PL intensity are found for all the investigated samples. Despite the loss of PL intensity increasing the temperature (Figure $6 \mathrm{c}$ ), the emission at room temperature is easily detectable. In order to correctly analyze the data, it is necessary to consider not only the variation with $T$ of the PL intensity, but also the change in the PL time evolution. Indeed, under the assumption that the overall dynamics of the states involved in the radiative recombination is linear at a given temperature $T$ (as proven by power-dependent measurements previously discussed), the time evolution of the PL intensity $I(E, t)$, at a given energy $E$, is proportional to:

$$
I(E, t) \propto \frac{N(E)}{\tau_{R}} \int_{0}^{t} S\left(t^{\prime}\right) F\left(E, t-t^{\prime}\right) d t^{\prime}
$$

where $S(t)$ represents the time evolution of the exciting laser pulse, or more generally the response function of the experimental setup, $N(E)$ the initial population of the excitonic 
states and $F(E, t)$ the function describing the PL time evolution normalized to 1 at $t=0$ (i.e., $F(E, 0)=1) \cdot \tau_{\mathrm{R}}$ is the radiative time that, as a consequence of the increasing population of the excitonic states outside the light cone, scales as $T^{3 / 2}$ in bulk samples [47]. If $S(t)$ is short enough with respect to the decay time, we can assume $S(t)=\delta(t)$ and then:

$$
I(E, t) \propto \frac{N(E) F(E, t)}{\tau_{R}}
$$
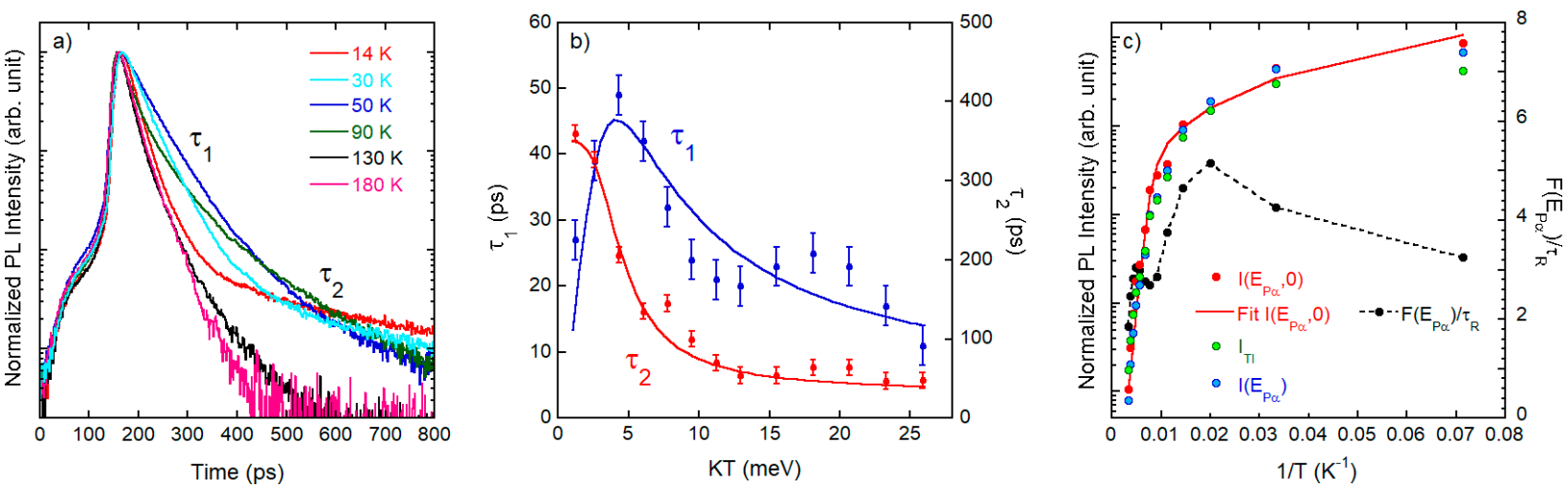

Figure 6. (a) PL time decay of $\mathrm{P}_{\alpha}$ at different temperatures over an extended time range. (b) Temperature dependence of $\tau_{1}$ and $\tau_{2}$ along with fits (solid lines). All data refers to sample A. (c) Arrhenius plots extracted from the PL intensity vs. $T$ along with the fit (solid line); $T$ dependence of the PL time evolution factor $F\left(E_{P \alpha}\right) / \tau_{\mathrm{R}}$ in linear scale. The dashed line is a guide for the eyes.

Therefore, in a TI measurement we get:

$$
I(E) \propto \frac{N(E) F(E)}{\tau_{R}}
$$

where $F(E)$ is the integral over $\mathrm{t}$, between 0 and infinite, of $F(E, t)$.

In general both $N(E)$ and $F(E, t)$ depend on temperature $(T)$ : as a consequence, from the Arrhenius plot of TI PL data we cannot distinguish between a quenching originating from $N(E)$, i.e., from a decrement of the initial filling of the state, or from $F(E, t)$, i.e., from a temperature evolution of radiative state dynamics.

In Figure $6 \mathrm{c}$ we report the Arrhenius plot of the PL intensity of the $\alpha$-band integrated in time and over all the spectrum (indicated as $I_{T I}$ ), of the TI PL intensity at $P_{\alpha}$ peak energy $E_{P \alpha}$ (i.e., $\left.I\left(E_{P \alpha}\right)=N\left(E_{P \alpha}\right) \times F\left(E_{P \alpha}\right) / \tau_{R}\right)$, and of the maximum of intensity at $E_{P \alpha}$ as obtained from the TR spectra at different temperatures (i.e., $\left.I\left(E_{P \alpha}, 0\right)=N\left(E_{P \alpha}\right) / \tau_{R}\right)$. On a linear scale we also plot the $T$-dependence of $F\left(E_{P \alpha}\right) / \tau_{R}$, as obtained from the TR PL at $E_{P \alpha}$ at the different temperatures.

The strong resemblance in the T-dependence of $I_{T I}$ and $I\left(E_{P \alpha}\right)$ brings evidence that the thermal quenching is the same for all the states responsible for the $\alpha$-band. Instead, the comparison between the Arrhenius plot of $I\left(E_{P \alpha}\right)$ and $I\left(E_{P \alpha}, 0\right)$ gives account of the different contributions to the thermal quenching: provided that the variation of $F\left(E_{P \alpha}\right) / \tau_{\mathrm{R}}$ with $T$ is quite small, it turns out that the main PL quenching comes from $N\left(E_{P \alpha}\right)$, i.e., from a decrease of the initial exciton population due to a fast (less than a few ps) free carriers capture in traps, before they reach the bottom of the band. In Figure $6 \mathrm{c}$ we show a fit of $I\left(E_{P \alpha}, 0\right)$ obtained with the expression:

$$
I\left(E_{P \alpha, 0}\right)=\frac{N\left(E_{P \alpha}\right)}{\tau_{R}}=\frac{A}{(k T)^{3 / 2}\left(1+B e^{-\frac{E_{A}}{k T}}\right)}
$$


where $E_{A}$ represents the effective activation energy of the capture center for the free carriers and the factor $(k T)^{3 / 2}$ accounts for the variation of $\tau_{\mathrm{R}}$ with $T$. In Figure $6 \mathrm{~b}$ we report $\tau_{1}$ and $\tau_{2}$ as obtained from the fits of the PL decay as a function of $T$, and the corresponding fits obtained with a standard two-level model describing the dynamics of an upper radiative state $\mathrm{U}$ interacting with a lower dark state L [48]. The fast time constant $\tau_{1}$ comes mainly from that of the upper state $U$ and is given by the parallel of a non-radiative $\left(\tau_{\mathrm{NR}}\right)$ and a radiative $\left(\tau_{R}\right)$ time constants. The non-radiative rate $1 / \tau_{\mathrm{NR}}$ is assumed to vary with $T$ as $\exp \left(-\mathrm{E}_{\mathrm{U}} / \mathrm{kT}\right)$ and the radiative rate $1 / \tau_{\mathrm{R}}$ to scale as $1 /(\mathrm{kT})^{3 / 2}$, according to data and theory for bulk systems [47]. The longer time constant $\tau_{2}$ is essentially the decay time of the lower state $L$, commonly attributed to a dark exciton [49], that thermally repopulates the upper one; the refilling rate from $L$ to $U$ is assumed to be thermally activated and then varying with $T$ as $\exp \left(-\mathrm{E}_{\mathrm{L}} / \mathrm{kT}\right)$. From the fit a quite long refilling time at low $T$ is obtained, i.e., $\approx 125 \mathrm{ps}$, and an activation energy $\mathrm{E}_{\mathrm{L}}=16 \mathrm{meV}$. For the activation energy of the non-radiative rate of the upper state we get from fit $\mathrm{E}_{\mathrm{U}}=7 \mathrm{meV}$, in agreement with the exciton localization energy $(\approx 7 \mathrm{meV})$. In other words, by increasing $T$, excitons are promoted from localized to extended states and then are quickly captured by non-radiative recombining centers. For the radiative rate of the upper level from the fit we get $\tau_{R}=40 \mathrm{ps}$ at low temperature that, taking into account the proper scaling with $T$, corresponds to $\tau_{\mathrm{R}}=450 \mathrm{ps}$ at room temperature, in agreement with literature [50,51]. Therefore, the strong decrease of the PL yield has to be ascribed, rather than to the exciton recombination, mainly to the reduced number of excitons that initially populate the radiative state: as a consequence, the presence of efficient traps for free carriers has to be invoked to explain the experimental findings as already found $[52,53]$. From this point of view, the observed presence of $\mathrm{O}$ and $\mathrm{C}$ in the XPS spectra (in particular $\mathrm{O}$ ) could be responsible for traps. The role of oxygen in halide perovskites (both hybrid and inorganic) has been investigated by several authors. On the one hand in hybrid perovskites oxygen promotes the material decomposition [54], while in $\mathrm{CsPbBr}_{3}$ it can produce passivation of surface states, that increases the PL yield [55], or, depending on the material nanostructure, a detrimental effect $[56,57]$. As an alternative, or in addition to this, also a $\mathrm{Pb}$ rich and a $\mathrm{Cl}$ defective surface can provide efficient non-radiative recombination [49,51].

In our samples, having measured the PL yield at room temperature, from the Arrhenius plot, we estimate a PL yield at $10 \mathrm{~K}$ of $\approx 90 \%$ which agrees with what expected from the radiative and non-radiative rate at low $T$ (Figure $6 \mathrm{~b}$ ).

\section{Conclusions}

We have demonstrated that fairly compact nanocrystalline thin films of $\mathrm{CsPCl}_{3}$ can be realized by RF magnetron sputtering with high homogeneity in terms of optical properties with at most a variation of the PL energy position of the order of $0.1 \%$ over $\sim 10 \mathrm{~cm}^{2}$. Samples prepared with this technique without any post-growth treatment and in absence of a polymeric coating show very good PL emission. To our knowledge, such homogeneity results are not achievable with standard deposition techniques without the addition of specific chemicals. In particular, the sample high optical quality is proven by the presence of limited IB $(\sim 15 \mathrm{meV})$. Detailed PL investigation provides quantitative information concerning the contribution of radiative and non-radiative recombination in $\mathrm{CsPbCl}_{3}$; the loss of PL yield comes from efficient capture of carriers in traps during the relaxation path. The presence of oxides could be responsible of such traps.

In conclusion, our results envisage the possibility of the use of RF magnetron sputtering for integration of fully inorganic perovskites in multilayer nanometric structures, such as photonic cavities, resonators and optical circuits.

Supplementary Materials: Additional experimental details and data concerning XPS, SEM, AFM and PL are available online at https:/ / www.mdpi.com/2079-4991/11/2/434/s1.

Author Contributions: Data curation, N.F., F.B. and A.V.; Formal analysis, N.F., N.C., G.R., A.R., F.B., C.B., M.B., S.C. and A.V.; Investigation, N.F., N.C., G.R., A.R., F.B., P.S., C.B., M.B. and A.V.; 
Supervision, A.V.; Writing—original draft, N.F.; Writing—review \& editing, N.C., F.B., P.S., M.B., S.C. and A.V. All authors have read and agreed to the published version of the manuscript.

Funding: This research was partially funded by Fondazione CR Firenze within the project "Supporto" (Bando Ricerca Scientifica 2019 call, project No. 24047) and within the project EPICO ("Eco-Perovskiti Inorganiche: Crescita e proprietà Optoelettroniche"; No. 2018.0950).

Data Availability Statement: The data presented in this study are available on request from the corresponding author.

Acknowledgments: The authors warmly acknowledge F. Bogani for helpful discussions and suggestions.

Conflicts of Interest: The authors declare no conflict of interest.

$\begin{array}{ll}\text { Abbreviations } \\ \text { PL } & \text { Photoluminescence } \\ \text { SLG } & \text { Soda Lime Glass } \\ \text { RF } & \text { Radio Frequency } \\ \text { SEM } & \text { Scanning Electron Microscopy } \\ \text { AFM } & \text { Atomic Force Microscopy } \\ \text { XPS } & \text { X-Ray Photoelectron Spectroscopy } \\ \text { XRD } & \text { X-Ray Diffraction } \\ \text { TI } & \text { Time-integrated } \\ \text { TR } & \text { Time-resolved } \\ \text { NC } & \text { Network of nanocrystals } \\ \text { MCs } & \text { Sub-micrometer size larger crystals } \\ \text { FE } & \text { Free Exciton } \\ \text { IB } & \text { Inhomogeneous Broadening }\end{array}$

\section{References}

1. Sutherland, B.R.; Sargent, E.H. Perovskite photonic sources. Nat. Photogr. 2016, 10, 295-302. [CrossRef]

2. Zhang, Y.; Lim, C.-K.; Dai, Z.; Yu, G.; Haus, J.W.; Zhang, H.; Prasad, P.N. Photonics and optoelectronics using nano-structured hybrid perovskite media and their optical cavities. Phys. Rep. 2019, 795, 1-51. [CrossRef]

3. Song, J.; Xu, L.; Li, J.; Xue, J.; Dong, Y.; Li, X.; Zeng, H. Monolayer and few-layer all-inorganic perovskites as a new family of two-dimensional semiconductors for printable optoelectronic devices. Adv. Mater. 2016, 28, 4861. [CrossRef]

4. Zhang, J.; Hodes, G.; Jin, S.; Liu, S. All-inorganic $\mathrm{CsPbX}_{3}$ perovskite solar cells: Progress and prospects. Angew. Chem. Int. Ed. 2019, 58, 15596. [CrossRef] [PubMed]

5. Bruzzi, M.; Talamonti, C.; Calisi, N.; Caporali, S.; Vinattieri, A. First proof-of-principle of inorganic perovskites clinical radiotherapy dosimeters. APL Mater. 2019, 7, 051101. [CrossRef]

6. Li, D.; Liao, P.; Shai, X.; Huang, W.; Liu, S.; Li, H.; Shena, Y.; Wang, M. Recent progress on stability issues of organic-inorganic hybrid lead perovskite-based solar cells. RSC Adv. 2016, 6, 89356. [CrossRef]

7. Mitzi, D.B. Synthesis, Structure, and Properties of Organic-Inorganic Perovskites and Related Materials, in Progress in Inorganic Chemistry; Karlin, K.D., Ed.; Wiley: Hoboken, NJ, USA, 1999; pp. 1-121.

8. Biccari, F.; Falsini, N.; Bruzzi, M.; Gabelloni, F.; Calisi, N.; Vinattieri, A. Defects in Perovskites for Solar Cells and LEDs, Chapter 3 in "Defects in Functional Materials"; Ling, F.C., Zhou, S., Kuznetsov, A., Eds.; World Scientific Publishing: Singapore, 2020; ISBN 978-9811203169. [CrossRef]

9. Protesescu, L.; Yakunin, S.; Bodnarchuk, M.I.; Krieg, F.; Caputo, R.; Hendon, C.H.; Yang, R.X.; Walsh, A.; Kovalenko, M.V. Nanocrystals of cesium lead halide perovskites $\left(\mathrm{CsPbX}_{3}, \mathrm{X}=\mathrm{Cl}, \mathrm{Br}\right.$, and I): Novel optoelectronic materials showing bright emission with wide color gamut. Nano Lett. 2015, 15, 3692. [CrossRef] [PubMed]

10. Yang, Z.; Zhang, S.; Li, L.; Chen, W. Research progress on large-area perovskite thin films and solar modules. J. Mater. 2017, 3, 231. [CrossRef]

11. Pasquarelli, R.M.; Ginley, D.S.; O’Hayre, R. Solution processing of transparent conductors: From flask to film. Chem. Soc. Rev. 2011, 40, 5406. [CrossRef] [PubMed]

12. Hirotsu, S.; Sawada, S. Crystal growth and phase transitions of $\mathrm{CsPbCl}_{3}$. Phys. Lett. 1969, 28, 762. [CrossRef]

13. Yunakova, N.; Miloslavsky, V.K.; Kovalenko, E.N.; Kovalenko, V.V. Effect of structural phase transitions on the exciton absorption spectrum of thin $\mathrm{CsPbCl}_{3}$ films. J. Low Temp. Phys. 2014, 40, 690. [CrossRef]

14. Heidrich, K.; Künzel, H.; Treusch, J. Optical properties and electronic structure of $\mathrm{CsPbCl}_{3}$ and $\mathrm{CsPbBr}_{3}$. Solid State Commun 1978, 25, 887. [CrossRef] 
15. Somma, F.; Aloe, P.; Lo Mastro, S. Structural and optical properties of ternary Cs-Pb-Cl nanoaggregates in thin films. J. Vac. Sci. Technol. 2001, 19, 2237. [CrossRef]

16. Nikl, M.; Nitsch, K.; Polak, K.; Pazzi, G.P.; Fabeni, P.; Citrin, D.S.; Gurioli, M. Optical properties of the Pb ${ }^{2+}$-based aggregated phase in a CsCl host crystal: Quantum-confinement effects. Phys. Rev. B 1995, 51, 5192. [CrossRef]

17. Kondo, S.; Nakagawa, H.; Saito, T.; Asada, H. Photoluminescence of $\mathrm{CsPbl}_{3}$ films prepared by quench deposition and subsequent heat treatments. J. Phys. Condens. Matt. 2003, 15, 1247. [CrossRef]

18. Borri, C.; Calisi, N.; Galvanetto, E.; Falsini, N. First proof-of-principle of inorganic lead halide perovskites deposition by magnetron-sputtering. Nanomaterials 2020, 10, 60. [CrossRef]

19. Scardi, P.; Setti, S.; Leoni, M. Multicapillary optics for materials science studies. Mat. Sci. Forum 2000, 321, 162. [CrossRef]

20. Yoshimura, Y.; Tozaki, K.; Kojima, A.; Iwasaki, H. In Smart Structures and Materials 2001: Active Materials: Behavior and Mechanics. In Proceedings of the SPIE 2001, Newport Beach, CA, USA, 4-8 March 2001; Volume 4333.

21. Iwanaga, M. Photoacoustic detection of phase transitions at low temperatures in $\mathrm{CsPbl}_{3}$ crystals. Phase Trans. 2005, 78, 377. [CrossRef]

22. Chen, H.; Guo, A.; Gu, X.; Feng, M. Highly luminescent $\mathrm{CsPbX}_{3}(\mathrm{X}=\mathrm{Cl}, \mathrm{Br}, \mathrm{I})$ perovskite nanocrystals with tunable photoluminescence properties. J. Alloys Comp. 2019, 789, 392. [CrossRef]

23. Moulder, J.F.; Stickle, W.F.; Sobol, P.E.; Bomben, K.D. Handbook of X-ray Photoelectron Spectroscopy; Perkin-Elmer Corporation: Eden Prairie, MI, USA, 1992.

24. Fröhlich, D.; Heidrich, K.; Künzel, H.; Trendel, G.; Treusch, J. Cesium-trihalogen-plumbates a new class of ionic semiconductors J. Luminescence 1979, 18, 385. [CrossRef]

25. Ito, H.; Onuki, H.; Onaka, R. Optical and photoelectronic studies of $\mathrm{CsPbCl}_{3}$ and $\mathrm{CsPbBr}_{3}$. J. Phys. Soc. Jpn. 1978, 45, 2043. [CrossRef]

26. Sebastian, M.; Peters, J.A.; Stoumpos, C.C.; Kostina, S.S.; Liu, Z.; Kanatzidis, M.G.; Freeman, A.J.; Wessels, B.W. Excitonic emissions and above-band-gap luminescence in the single-crystal perovskite semiconductors $\mathrm{CsPbBr}_{3}$ and $\mathrm{CsPbCl}_{3}$. Phys. Rev. B 2015, 92, 235210. [CrossRef]

27. Pashuk, P.; Pidzyrailo, N.S.; Matsko, M.G. Exciton absorption, luminescence, and resonant Raman scattering in $\mathrm{CsPbCl}_{3}$ and $\mathrm{CsPBr}_{3}$ at low temperatures. Sov. Phys. Solid State 1981, 23, 1263.

28. Lohar, A.; Shinde, A.; Gahlaut, R.; Sagdeo, A.; Mahamuni, S. Enhanced photoluminescence and stimulated emission in $\mathrm{CsPCl}_{3}$ nanocrystals at low temperature. J. Phys. Chem. C 2018, 122, 25014. [CrossRef]

29. Ito, H.; Nakahara, J.; Onaka, R. Magneto-optical study of the exciton states in CsPbCl 3 . J. Phys. Soc. Jpn. 1979, 47, 1927. [CrossRef]

30. Becker, M.A.; Scarpelli, L.; Nedelcu, G.; Rainò, G.; Masia, F.; Borri, P.; Stöferle, T.; Kovalenko, M.V.; Langbein, W.; Mahrt, R.F. Long exciton dephasing time and coherent phonon coupling in $\mathrm{CsPbr}_{2} \mathrm{Cl}$ perovskite nanocrystals. Nano Lett. 2018, $18,7546$. [CrossRef] [PubMed]

31. Shah, J. Ultrafast Spectroscopy of Semiconductors and Semiconductor Nanostructures; Springer: Heidelberg, Germany, 1996.

32. Gabelloni, F.; Biccari, F.; Falsini, N.; Calisi, N.; Caporali, S.; Vinattieri, A. Long-living nonlinear behavior in $\mathrm{CsPbBr}_{3}$ carrier recombination dynamics. Nanophotonics 2019, 8, 1447. [CrossRef]

33. Cavigli, L.; Bogani, F.; Vinattieri, A.; Faso, V.; Baldi, G. Volume versus surface-mediated recombination in anatase TiO 2 nanoparticles. J. Appl. Phys. 2009, 106, 053516. [CrossRef]

34. Kondo, S.; Suzuki, K.; Saito, T.; Asada, H.; Nakagawa, H. Confinement-enhanced stimulated emission in microcrystalline $\mathrm{CsPCl}_{3}$ films grown from the amorphous phase. J. Cryst. Growth 2005, 282, 94. [CrossRef]

35. Nikl, M.; Birch, D.J.S.; Polak, K. Blue and violet emission of $\mathrm{PbCl}_{2}$. Phys. Solid State 1991, 165, 611. [CrossRef]

36. Voloshinovskii, A.; Myagkota, S.; Gloskovskii, A.; Zazubovich, S. Luminescence of $\mathrm{CsPbCl}_{3}$ nanocrystals dispersed in a CsCl crystal under high-energy excitation. Phys. Solid State 2001, 225, 257. [CrossRef]

37. Bai, K.; Tan, R.; Ke, B.; Xue, X.; Zhao, J.; Zouc, B.; Zeng, R. Room temperature synthesis of Mn-doped $\mathrm{Cs}_{3} \mathrm{~Pb}_{6.48} \mathrm{Cl}_{16} \mathrm{Perovskite}$ nanocrystals with pure dopant emission and temperature-dependent photoluminescence. Cryst. Eng. Comm. 2019, 21, 3568-3575. [CrossRef]

38. Biccari, F.; Gabelloni, F.; Burzi, E.; Gurioli, M.; Pescetelli, S.; Agresti, A.; Castillo, A.E.D.; Ansaldo, A.; Kymakis, E.; Bonaccorso, F.; et al. Graphene-based electron transport layers in perovskite solar cells: A step-up for an efficient carrier collection. Adv. Energy Mat. 2017, 7, 1701349. [CrossRef]

39. Dobrovolsky, A.; Merdasa, A.; Unger, E.L.; Yartsev, A.; Scheblykin, I.G. Defect-induced local variation of crystal phase transition temperature in metal-halide perovskites. Nat. Commun. 2017, 8, 34. [CrossRef]

40. Meng, X.; Zhang, R.; Fu, Z.; Zhang, Q. Domain-dependent electronic structure and optical absorption property in hybrid organic-inorganic perovskite. Phys. Chem. Chem. Phys. 2016, 18, 27358. [CrossRef] [PubMed]

41. Piveteau, L.; Aebli, M.; Yazdani, N.; Millen, M.; Korosec, L.; Krieg, F.; Benin, B.M.; Morad, V.; Piveteau, C.; Shiroka, T.; et al. Bulk and nanocrystalline cesium lead-halide perovskites as seen by halide magnetic resonance. ACS Cent. Sci. 2020, 6, 1138-1149. [CrossRef] [PubMed]

42. Shi, H.; Zhang, X.; Sun, X.; Zhang, X. Phonon mode transformation in size-evolved solution-processed inorganic lead halide perovskite. Nanoscale 2018, 10, 9892. [CrossRef]

43. Cape, J.A.; White, R.L.; Feigelson, R.S. EPR study of the structure of CsPbCl $\mathrm{Cl}_{3}$. J. Appl. Phys. 1969, 40, 5001. [CrossRef] 
44. Carabatos-Nédelec, C.; Oussaïd, M.; Nitsch, K. Raman scattering investigation of cesium plumbochloride, $\mathrm{CsPbCl}_{3}, \mathrm{phase}$ transitions. J. Raman Spectrosc. 2003, 34, 388. [CrossRef]

45. Yi, J.; Ge, X.; Liu, E.; Cai, T.; Zhao, C.; Wen, S.; Sanabria, H.; Chen, O.; Rao, A.M.; Gao, J. The correlation between phase transition and photoluminescence properties of $\mathrm{CsPbX}_{3}(\mathrm{X}=\mathrm{Cl}, \mathrm{Br}, \mathrm{I})$ perovskite nanocrystals. Nanoscale Adv. 2020, 2, 4390. [CrossRef]

46. Becker, M.; Vaxenburg, R.; Nedelcu, G.; Sercel, P.; Shabaev, A.; Mehl, M.; Michopoulos, J.; Lambrakos, S.; Bernstein, N.; Lyons, J.; et al. Bright triplet excitons in caesium lead halide perovskites. Nature 2018, 553, 189-193. [CrossRef] [PubMed]

47. Andreani, L.C.; d'Andrea, A.; del Sole, R. Excitons in confined systems: From quantum well to bulk behaviour. Phys. Lett. A 1992, 168, 451. [CrossRef]

48. Haug, R. (Ed.) Advances in Solid State Physics; Springer: New York, NY, USA, 2008; Volume 48.

49. Chen, L.; Li, B.; Zhang, C.; Huang, X.; Wang, X.; Xiao, M. Composition-dependent energy splitting between bright and dark excitons in lead halide perovskite nanocrystals. Nano Lett. 2018, 18, 2074. [CrossRef]

50. Mondal, N.; De Samanta, A. Achieving near-unity photoluminescence efficiency for blue-violet-emitting perovskite nanocrystals. ACS Energy Lett. 2019, 4, 32. [CrossRef]

51. Ahmed, G.H.; El-Demellawi, J.K.; Yin, J.; Pan, J.; Velusamy, D.B.; Hedhili, M.N.; Alarousu, E.; Bakr, O.M.; Alshareef, H.N.; Mohammed, O.F. Giant photoluminescence enhancement in $\mathrm{CsPbCl}_{3}$ perovskite nanocrystals by simultaneous dual-surface passivation. ACS Energy Lett. 2018, 3, 2301. [CrossRef]

52. Ahumada-Lazo, R.; Alanis, J.A.; Parkinson, P.; Binks, D.J.; Hardman, S.J.O.; Griffiths, J.T.; Rivarola, F.W.R.; Humphrey, C.J.; Ducati, C.; Davis, N.J.L.K. Emission properties and ultrafast carrier dynamics of $\mathrm{CsPbCl}_{3}$ perovskite nanocrystals. J. Phys. Chem. C 2019, 123, 2651. [CrossRef]

53. Lai, R.; $\mathrm{Wu}, \mathrm{K}$. Picosecond electron trapping limits the emissivity of $\mathrm{CsPbCl}_{3}$ perovskite nanocrystals. J. Chem. Phys. 2019, 151, 194701. [CrossRef]

54. Aristidou, N.; Sanchez-Molina, I.; Chotchuangchutchaval, T.; Brown, M.; Martinez, L.; Rath, T.; Haque, S.A. The role of oxygen in the degradation of methylammonium lead trihalide perovskite photoactive layers. Angew. Chem. Int. Ed. 2015, 54, 8208-8212. [CrossRef] [PubMed]

55. Lu, D.; Zhang, Y.; Lai, M.; Xie, C.; Lin, J.; Lei, T.; Lin, Z.; Kley, C.S.; Huang, J.; Rabani, E.; et al. Giant light-emission enhancement in lead halide perovskites by surface oxygen passivation. Nano Lett. 2018, 18, 6967-6973. [CrossRef]

56. Rodà, C.; Abdelhady, A.L.; Shamsi, J.; Lorenzon, M.; Pinchetti, V.; Gandini, M.; Meinardi, F.; Manna, L.; Brovelli, S. O 2 as a molecular probe for nonradiative surface defects in $\mathrm{CsPbr}_{3}$ perovskite nanostructures and single crystals. Nanoscale 2019, 11, 7613. [CrossRef]

57. Meggiolaro, D.; Mosconi, E.; De Angelis, F. Mechanism of reversible trap passivation by molecular oxygen in lead-halide perovskites. ACS Energy Lett. 2017, 2, 2794-2798. [CrossRef] 